\title{
Measuring achievement and shortfall improvements in a consistent way
}

\author{
Iñaki Permanyer \\ Centre d'Estudis Demogràfics \\ Edifici E-2, Campus de la UAB \\ E-mail: inaki.permanyer@uab.es \\ Phone: +34 935813060
}

\begin{abstract}
When standards of living are measured with bounded indicators-as is commonly the case with health or education variables-improvements over time can be assessed through achievements or the corresponding shortfalls. Integrating both approaches into a common framework, we propose the corresponding achievement and shortfall improvement indices and characterize them axiomatically. However, rankings of alternative social states in terms of achievements and shortfalls do not necessarily go hand in hand. We present the necessary and sufficient conditions under which our achievement and shortfall improvement indices rank social states in a consistent way. Since improvement indices proposed in the literature so far have only shown results at the country level, we suggest collecting information at arbitrary small levels of aggregation to be able to uncover unobserved heterogeneity, complementing traditional country-level analysis and taking into account the entire distribution of local-level improvements over time. Empirical illustrations using census data from five African countries suggest that consistency between achievement and shortfall improvements in standards of living is not only a matter of theoretical import but it is also a problem that can be encountered in practice to a large extent.
\end{abstract}

Keywords: Improvement measurement, Bounded variables, Shortfall, Achievement, Consistency, Inequality aversion.

JEL Codes: D63, I31 


\section{Introduction}

When assessing the standard of living of our societies, it is important to look not only at the prevailing levels of different key socio-economic and development indicators but also to their changes over time. While several studies have explored the dynamics of well-being (e.g.: Dasgupta and Weale, 1992; Dasgupta, 1993; Easterly, 1999; Mazumdar, 1999), only a few of them have attempted to define "improvement" or "progress" measures in a rigorous and satisfactory way - an important issue that, as will be argued below, has received insufficient attention from scholars. The only standard of living improvement measures proposed so far we are aware of are those of Kakwani (1993), which were axiomatically characterized a few years later by Majumder and Chakravarty (1996) and extended to the multidimensional framework by Tsui (1996) and Chakravarty and Mukherjee (1999). Unfortunately, these measures do not address a couple of relevant matters that have been ignored so far in the literature and which will be the main concern of this paper: the problem of "consistently measuring achievement and shortfall improvement" and the problem of "unobserved heterogeneity".

Given the bounded nature of virtually all indices of standard of living (typical examples include health or education variables like life expectancy, child or adult mortality rates, literacy or school attendance rates, educational attainment and so forth), it is a priori possible to focus on the distribution of achievements or on the corresponding distribution of shortfalls with respect to the upper bound when measuring improvements over time. To illustrate: improvements in the coverage of public health plans could be assessed via the percentage of vaccinated children (an achievement indicator) or through the percentage of unvaccinated children (a shortfall indicator). While both approaches seem attractive on their own right, 
the few formal attempts to measure improvements in standards of living we are aware of have - somewhat strangely - only focused on the changes in the shortfall distributions (see Kakwani 1993, Majumder and Chakravarty 1996, Tsui 1996 and Chakravarty and Mukherjee 1999). However, we see a priori no reason to focus exclusively on those distributions and disregard their achievement counterparts. Apparently, both perspectives offer complementary views of the same problem, so it seems important to assess them in relation to each other. In this context, a natural question that might arise is: will improvements in shortfalls and improvements in achievements mirror each other or not? In this paper we will consider these complementary approaches simultaneously and present the conditions under which both classes of measures rank alternative states of affairs in a consistent way.

In the last few years, there has been a bourgeoning debate on the consistent measurement of achievement and shortfall inequality for bounded variables. The potential (and actual) mismatch between certain achievement and shortfall inequality measures was signaled by Clarke et al. (2002) and several authors have attempted to overcome that problem (see Erreygers 2009, Lambert and Zheng 2011 and Lasso de la Vega and Aristondo 2012). Even if some of the results presented in this paper are inspired in the aforementioned works, there are important differences that are worth pointing out. On the one hand, we are not dealing with inequality but with improvements over time, so the functional forms of the indices we will be working with are completely different. On the other hand, while the notion of "inequality" is the same regardless of whether we are considering achievements or shortfalls (i.e.: in both cases we are measuring the spread of a set of numbers), the notion of "improvement" can be considered as being, so to say, directional (as it depends on the end from which one stares at it), so it is important to distinguish between the two perspectives. Hence, while the same inequality index is used to measure attainment and shortfall inequality 
(the only thing that changes is the domain of the inequality index), we will need to define a specific improvement function for achievements and a specific improvement function for shortfalls. As a consequence, the conditions that are needed to satisfy the attainmentshortfall consistency test presented in this paper will differ with respect to the conditions used in the aforementioned papers.

Another important issue that has not been addressed in former attempts to measure improvements in standard of living is the problem of unobserved heterogeneity. Since the indices proposed so far are designed to measure improvements experienced at the country level, they are only showing highly aggregated average values that might actually hide enormous internal inequalities. It is in this context that this paper also aims to make a contribution: collecting information on the standard of living at arbitrary small levels of aggregation (e.g.: province, municipality, individual) we are able to unravel local patterns of improvement that are not discernible to "classical" (i.e.: country level) approaches. We contend that such finely grained information can be used in at least two complementary ways. On the one hand one can explore the distribution of improvements as is, offering the possibility to researchers and policy-makers of investigating local level improvement patterns and their relationship with key socio-economic or demographic variables. This approach is conceptually related to recent attempts of constructing subgroup specific versions of welfare indices that were originally defined at the country level (as is the case with the Human Development Index, which has been redefined for income quintiles, migrants and non-migrants, households or different administrative units; see Grimm, Harttgen, Klasen and Misselhorn 2008, Grimm et al. 2010, Harttgen and Klasen 2011a, 2011b, Permanyer 2013). On the other hand, one might attempt to summarize that wealth of information into an overall improvement index that takes into account certain characteristics of the underlying distribution (e.g.: inequality). This second 
route is reminiscent of other recent attempts to construct subgroup-consistent, inequality and/or association-sensitive and nationally representative human development indices (see Alkire and Foster 2010, Foster et al 2005, Seth 2009). In this paper, we illustrate different ways in which both approaches can be operationalized.

The finely-grained perspective suggested here brings to the fore distributional issues that have not been explicitly incorporated so far in the literature on the measurement of improvements in standard of living. In this respect, the monitoring of global campaigns like the United Nations' Millennium Development Goals could benefit enormously from it since the reports presented so far (e.g.: UN 2010a,b,c,d,e) deal only with country-level variations in standards of living, leaving aside the variations that might take place at local or regional levels. In order to illustrate the usefulness of our proposal, we present the evolution over time of child health outcomes -which are highly related to MDG \#4 (reduce child mortality)- at low aggregation levels for five African countries using census microdata from the Integrated Public Use Microdata Series (IPUMS) project.

The rest of the paper is organized as follows. In section 2, we present the main axioms and characterize the improvement measures used in the paper. In section 3, we explore the problem of consistently measuring achievement and shortfall improvements. Section 4 shows the empirical illustration and Section 5 concludes. The proofs are relegated to the appendix.

\section{Axioms and improvement measures}

We consider a population partitioned in $n \in \mathbb{N}$ units of analysis $\{1, \ldots, n\}$. The precise definition of these units of analysis will depend on the specific context one is working with. For instance, one might be interested in tracking changes in standards of living for individu- 
als, households, neighborhoods, municipalities and so on. For ease of notation, the units of analysis will often be simply referred to as 'individuals', even if in practice one might actually work with households, neighborhoods, municipalities or any other population subgroup of size $w_{i}$. The achievement level of individual $i$ will be measured with a certain standard of living indicator that will be tracked in two different moments in time (say, $T_{1}$ and $T_{2}$, with $T_{1}<T_{2}$ ). We assume that such indicator is measured in a positive scale - an almost universal assumption in standards of living or well-being measurement - and that its values are naturally bounded from above and below. This last assumption is very common for most indicators that are typically incorporated in standard of living or well-being assessments. For instance: health or education variables (like life expectancy, mortality rates, educational attainment) can not increase or decrease indefinitely, so it is highly plausible to place a lower and upper bound on them. In this paper, the lower and upper bounds will simply be denoted by $L$ and $U$ respectively, with $0 \leq L<U$. It might be worth emphasizing that we are assuming that our achievement indicators can actually attain the values of the lower and upper bounds $L, U$. This is in contrast with the approach followed in other conceptually related studies, where the upper bound $U$ is assumed to be unattainable (see Kakwani 1993, Majumder and Chakravarty 1996 and Tsui 1996). Our choice has been motivated by the fact that many variables typically included in the assessment of the standard of living (e.g.: literacy rates, enrolment ratios, gender gaps and so on) do very often reach their upper bounds ${ }^{1}$. This apparently minor technicality has important consequences in the derivation of the functional form of our improvement indices.

We start our analysis exploring the single individual case (i.e.: $n=1$ ).

${ }^{1}$ In case the underlying variable does not reach the upper bound (for instance, in the case of life expectancy), the results presented in this paper are equally valid. 


\subsection{Individual improvements}

We will denote by $x \in[L, U]$ the achievement level of a given unit of analysis in time $T_{1}$. Analogously, we will denote by $y \in[L, U]$ the achievement level of the same unit of analysis in time $T_{2}$. In this context, we can naturally define the shortfalls associated to achievements $x$ and $y$ as $p:=U-x$ and $q:=U-y$. Clearly, $p, q \in[0, U-L]$. When it comes to measure the notion of "improvement", we should first decide whether the later will be assessed through changes in achievements or in shortfalls. In this respect, the few formal attempts to measure improvements in standards of living we are aware of have only focused on the changes in shortfalls (see Kakwani 1993, Majumder and Chakravarty 1996, Tsui 1996 and Chakravarty and Mukherjee 1999). The fact that the corresponding achievements are disregarded in those papers is somewhat surprising, particularly because the existence of both approaches is acknowledged from the start. Since we consider that changes in both achievements and shortfalls are essentially measuring two sides of the same coin, in this paper we will incorporate them simultaneously and show the conditions under which they rank alternative states of affairs in a consistent way. When improvements in standards of living are assessed through changes in achievements, we will introduce a so-called "achievement improvement index". Formally: an achievement improvement index $\iota^{a}$ is defined as a nontrivial real-valued function $\iota^{a}:([L, U] \times[L, U]) \rightarrow \mathbb{R}$. The values of $\iota^{a}(x, y)$ should be interpreted as the improvement in standard of living of a given unit of analysis when the corresponding achievement changes from $x$ to $y$. Analogously, a shortfall improvement index $\iota^{s}$ is defined as a non-trivial real-valued function $\iota^{s}:([0, U-L] \times[0, U-L]) \rightarrow \mathbb{R}$ and its values $\iota^{s}(p, q)$ should also be interpreted as the improvement in standard of living observed when the shortfall changes from $p$ to $q$.

Interestingly, the fact of introducing different indices to measure achievement and short- 
fall improvements (i.e.: $\iota^{a}$ and $\iota^{s}$ ) is in contrast with the approach followed in the measurement of achievement and shortfall inequality. In the later case, the same inequality index $D$ is used to measure both concepts, the only thing that changes is the domain (i.e.: one compares inequality of a distribution of achievements $D\left(x_{1}, \ldots, x_{n}\right)$ vis-à-vis inequality of the corresponding distribution of shortfalls $\left.D\left(U-x_{1}, \ldots, U-x_{n}\right)\right)$. As mentioned in the introduction, the notion of "inequality" is the same regardless of whether we are considering one distribution or the other but the notion of "improvement" depends on whether we use achievements or shortfalls.

Given the fact that the achievement and shortfall improvement measures are based on the same underlying ideas, our axioms will be presented in the following way. We will first present the general intuition behind the corresponding axiom and then formally show how this idea translates into certain restrictions for the $\iota^{a}$ and $\iota^{s}$ functions separately. Our first axiom reads as follows.

Continuity $(\mathrm{CN})$ : The improvement indices are continuous functions, that is: $\iota^{a}$ and $\iota^{s}$ are continuous.

This is an extremely common assumption in the literature of socio-economic indices. It requires that small changes in the achievements or shortfalls of individuals produce small changes in the corresponding improvement function. Stated otherwise: the change in standard of living does not abruptly change as individuals' achievements or shortfalls are slightly altered. Among other things, this property ensures that our measures will not be dramatically affected by measurement errors.

Monotonicity (MN): When a given unit of analysis sees its standard of living increasing from $T_{1}$ and $T_{2}$, then the corresponding improvement index should increase. In other words, 
$\iota^{a}(x, y)$ is increasing in $y$ and $\iota^{s}(p, q)$ is decreasing in $q$.

This assumption is quite unexceptionable for any index attempting to measure improvements in standard of living between any two moments in time.

Homotheticity (HM): When the variable that is used to measure the standard of living (either in terms of achievements or shortfalls) is scaled by a positive constant, the ranking between two alternative states of affairs in terms of improvement should remain unaltered. Formally: for all $x_{1}, y_{1}, x_{2}, y_{2} \in[L, U]$ and all $\lambda>0$ such that $\lambda x_{1}, \lambda y_{1}, \lambda x_{2}, \lambda y_{2} \in[L, U]$, one has that

$$
\iota^{a}\left(x_{1}, y_{1}\right) \geq \iota^{a}\left(x_{2}, y_{2}\right) \Leftrightarrow \iota^{a}\left(\lambda x_{1}, \lambda y_{1}\right) \geq \iota^{a}\left(\lambda x_{2}, \lambda y_{2}\right)
$$

Analogously for $\iota^{s}:$ for all $p_{1}, q_{1}, p_{2}, q_{2} \in[0, U-L]$ and all $\widetilde{\lambda}>0$ such that $\tilde{\lambda} p_{1}, \tilde{\lambda} q_{1}, \widetilde{\lambda} p_{2}, \tilde{\lambda} q_{2} \in$ $[0, U-L]$, one has that

$$
\iota^{s}\left(p_{1}, q_{1}\right) \geq \iota^{s}\left(p_{2}, q_{2}\right) \Leftrightarrow \iota^{s}\left(\widetilde{\lambda} p_{1}, \widetilde{\lambda} q_{1}\right) \geq \iota^{s}\left(\widetilde{\lambda} p_{2}, \widetilde{\lambda} q_{2}\right)
$$

Homotheticity essentially requires that our rankings in terms of improvements are not affected by the scale of measurement used in our standard of living indicators.

Upward Sensitivity (US): Other things being equal, an improvement index should reward those improvements occuring at higher achievement levels. Formally: consider a hypothetical scenario with $L \leq z<w \leq U$. Take now any $\delta>0$ such that $z+\delta, w+\delta \in[L, U]$. Then one has that

$$
\iota^{a}(z, z+\delta) \leq \iota^{a}(w, w+\delta)
$$

Analogously for $\iota^{s}$ : consider a hypothetical scenario with $0 \leq z<w \leq U-L$. Take now any $\delta>0$ such that $z-\delta, w-\delta \in[0, U-L]$. Then one has that

$$
\iota^{s}(z, z-\delta) \geq \iota^{s}(w, w-\delta)
$$


Different authors have argued that, for certain standard of living indicators, improvement is much more difficult as the achievement level of the attribute becomes higher and higher (e.g.: Sen 1981, 1992, Dasgupta 1993). Sen (1981), for instance, argues that it is not the same to increase life expectancy from 40 to 45 years than increasing it from 75 to 80 . In this line, Upward Sensitivity states that an increase of $\delta$ units in our improvement indicator is to be more valued when the initial achievement level is higher. This property has also been incorporated in all other improvement indices proposed in the literature so far.

Weak Additivity (WA): The addition of the improvement in living standards from $T_{1}$ to $T_{2}$ and the improvement in living standards from $T_{2}$ to $T_{3}$ only depends on the initial and the final achievement or shortfall levels. Formally: consider $x, y, z \in[L, U]$. Then

$$
\iota^{a}(x, y)+\iota^{a}(y, z)=\varphi(x, z)
$$

for some function $\varphi:[L, U]^{2} \rightarrow \mathbb{R}$. Analogously for $\iota^{s}$ : consider $p, q, r \in[0, U-L]$. Then

$$
\iota^{s}(p, q)+\iota^{s}(q, r)=\phi(p, r)
$$

for some function $\phi:[0, U-L]^{2} \rightarrow \mathbb{R}$.

According to Weak Additivity, the evaluation of the changes in living standards between two moments in time depends exclusively on these two moments in time and is not affected by the intermediate changes that might have occurred in between. Interestingly, this axiom is less stringent than 'Additivity', a somewhat restrictive axiom introduced by Kakwani (1993), Majumder and Chakravarty (1996), Tsui (1996) and Chakravarty and Mukherjee $(1999)^{2}$ stating that for any three periods $T_{1}, T_{2}, T_{3}$, the change from period $T_{1}$ to period $T_{3}$ can be expressed exactly as the sum of the change from period $T_{1}$ to period $T_{2}$ and that

2 In those papers the same axiom is named using alternative labels (e.g.: 'Subperiod Consistency' or 'Period Consistency'). 
from period $T_{2}$ to period $T_{3}$ (that is: $\iota^{a}(x, y)+\iota^{a}(y, z)=\iota^{a}(x, z)$ for all $x, y, z \in[L, U]$ and $\iota^{s}(p, q)+\iota^{s}(q, r)=\iota^{s}(p, r)$ for all $\left.p, q, r \in[0, U-L]\right)$. Relaxing the Additivity axiom in favor of its weaker version we enlarge the class of admissible indices to include other improvement measures that are interesting for the purposes of this paper. Clearly, Additivity implies Weak Additivity but the opposite is not true.

Normalization (NM): In order to render results easily interpretable, it is customary to normalize the values of our improvement indicators between well-known bounds. Formally: $\iota^{a}(U, L)=\iota^{s}(0, U-L)=A$ and $\iota^{a}(L, U)=\iota^{s}(U-L, 0)=B$ for some real constants $A<B$.

Normalization stipulates that the improvement functions for a society with $(n=) 1$ individual take their maximal value - equal to $B$ - whenever the achievement indicator starts in its lowest level in $T_{1}$ and ends up at its highest level in $T_{2}$. NM also stipulates that whenever the achievement indicator goes from its highest level in $T_{1}$ and ends up at its lowest level in $T_{2}$, then the improvement index should take a value of $A$. While somewhat arbitrary, the practice of bounding the values of socio-economic indicators into a certain range $[A, B]$ is extremely extended in the literature. Standard and simple choices for such bounds could be $[-1,1],[-100,100],[0,1]$ or $[0,100]$.

Combining these different axioms, we are able to characterize our achievement and shortfall improvement indices.

Theorem 1. An achievement improvement index $\iota^{a}$ satisfies the "achievement version" of the axioms CN, MN, HM, US, WA and NM if and only if it can be written as

$$
\iota^{a}(x, y)=\frac{B-A}{2\left(U^{\alpha}-L^{\alpha}\right)}\left[y^{\alpha}-x^{\alpha}\right]+\frac{A+B}{2}
$$

for all $x, y \in[L, U]$ and for some real parameters $\alpha \geq 1, A<B$. Analogously, a shortfall improvement index $\iota^{s}$ satisfies the "shortfall version" of those axioms if and only if it can be 
written as

$$
\iota^{s}(p, q)=\frac{B-A}{2(U-L)^{\beta}}\left[p^{\beta}-q^{\beta}\right]+\frac{A+B}{2}
$$

for all $p, q \in[0, U-L]$ and for some real parameters $\beta \in(0,1], A<B$.

Proof: See the Appendix.

Remark 1. Interpretation of the indices. By construction, the values of $\iota^{a}$ and $\iota^{s}$ range between $A$ and $B$. When an individual starts at the lowest possible achievement level in $T_{1}$ (i.e.: $L$ ) and ends up at the highest possible achievement level in $T_{2}$ (i.e.: $U$ ), then both $\iota^{a}$ and $\iota^{s}$ take a value of $B$. Alternatively, when an individual starts at the highest possible achievement level in $T_{1}$ and ends up at the lowest possible achievement level in $T_{2}$, then both $\iota^{a}$ and $\iota^{s}$ take a value of $A$. When no change at all is observed between times $T_{1}$ and $T_{2}$, both $\iota^{a}$ and $\iota^{s}$ take a value of $(A+B) / 2$. Therefore, values of $\iota^{a}$ and $\iota^{s}$ above (resp. below) $(A+B) / 2$ should be interpreted as an improvement (resp. worsening) in the standard of living of the corresponding unit of analysis between times $T_{1}$ and $T_{2}$. As can be seen, even if both indices $\iota^{a}$ and $\iota^{s}$ are highly related and have much in common - they are basically focusing on complementary aspects of the same phenomenon - their respective functional forms have some essential differences and they can not be derived from one another via "simple" (e.g.: monotonic) transformations.

Remark 2. Relationship with other measures. To our knowledge, the improvement indices characterized in Theorem 1 are the first measures of their kind that explicitly incorporate the achievement and shortfall perspectives in a common framework. When choosing $A=-1$ and $B=1$, our shortfall improvement index $\iota^{s}$ partly coincides with the improve- 
ment indices suggested by Kakwani (1993:314), which can be written as follows:

$$
f(U-x, U-y):=\left\{\begin{array}{c}
\frac{(U-x)^{r}-(U-y)^{r}}{(U-L)^{r}} \text { if } 0<r<1 \\
\frac{\ln (U-x)-\ln (U-y)}{\ln (U-L)} \text { if } r=0
\end{array}\right\}=\left\{\begin{array}{c}
\frac{p^{r}-q^{r}}{(U-L)^{r}} \text { if } 0<r<1 \\
\frac{\ln (p)-\ln (q)}{\ln (U-L)} \text { if } r=0
\end{array}\right\}=f(p, q)
$$

Theorem 1 characterizes axiomatically Kakwani's improvement index $f$ in a complementary way that differs from the characterization presented in Majumder and Chakravarty (1996). However, note that the logarithmic functional form appearing in equation (9) does not appear in equation (8) because we are assuming that the bounds of the underlying indicator's domain are attainable ${ }^{3}$.

Remark 3. Interpretation of parameters. Parameters $\alpha$ and $\beta$ regulate the extent to which improvements at higher achievement levels are given more importance or not for the achievement and shortfall indices respectively (i.e.: they regulate whether $\iota^{a}$ and $\iota^{s}$ comply with the Upward Sensitivity axiom or not). The farther away these parameters are from the value of 1 (with $\alpha>1$ and $\beta \in(0,1)$ ), the more our measures will reward those improvements occuring at higher achievement or lower shortfall levels. At the other extreme, when $\alpha=\beta=1$, our measures will not be sensitive to the difficulty of further improvement at higher achievement levels. Interestingly, when $\alpha$ and $\beta$ coincide at 1 , it turns out that $\iota^{a}(x, y)$ and $\iota^{s}(p, q)$ are exactly the same.

\subsection{The multi-individual case: introducing heterogeneity}

In the previous section we defined attainment and shortfall improvement indices for a single unit of analysis. We are now going to extend those ideas to the case where we have $n$ units of analysis of sizes $\left(w_{1}, \ldots, w_{n}\right)$. We will denote by $\mathbf{x}=\left(x_{1}, \ldots, x_{n}\right) \in[L, U]^{n}$ the achievement

3 If the upper bound $U$ were assumed to be unattainable, then the characterization in Theorem 1 would also produce the logarithmic form appearing in equation (9). 
distribution of the population in time $T_{1}$, where $x_{i}$ represents the achievement of individual $i$ in $T_{1}$. Analogously, we will denote by $\mathbf{y}=\left(y_{1}, \ldots, y_{n}\right) \in[L, U]^{n}$ the achievement distribution of the population in time $T_{2}$, where $y_{i}$ represents the achievement of individual $i$ in $T_{2}$. In this context, we can naturally define the shortfall distributions of the population in times $T_{1}$ and $T_{2}$ associated to $\mathbf{x}$ and $\mathbf{y}$ as $\mathbf{p}=\left(p_{1}, \ldots, p_{n}\right):=\left(U-x_{1}, \ldots, U-x_{n}\right) \in[0, U-L]^{n}$ and $\mathbf{q}=\left(q_{1}, \ldots, q_{n}\right):=\left(U-y_{1}, \ldots, U-y_{n}\right) \in[0, U-L]^{n}$ respectively. When no confusion arises, these shortfall distributions might also be denoted as $U-\mathbf{x}$ and $U-\mathbf{y}$ respectively. Applying the indices characterized in Theorem 1 to the achievement and shortfall values of each individual we obtain the corresponding distribution of improvements. In case of achievements, this is $\boldsymbol{\iota}^{a}(\mathbf{x}, \mathbf{y}):=\left(\iota^{a}\left(x_{1}, y_{1}\right), \ldots, \iota^{a}\left(x_{n}, y_{n}\right)\right)$ and for shortfalls we have $\iota^{s}(\mathbf{p}, \mathbf{q}):=\left(\iota^{s}\left(p_{1}, q_{1}\right), \ldots, \iota^{s}\left(p_{n}, q_{n}\right)\right)$. Since the ideas introduced in this section are applicable to both distributions indistinctly, we will generally speak about "the" improvements distribution $\left(\iota_{1}, \ldots, \iota_{n}\right)$, where $\iota_{i}$ can either be $\iota^{a}\left(x_{i}, y_{i}\right)$ or $\iota^{s}\left(p_{i}, q_{i}\right)$.

The fact of having an improvements distribution $\left(\iota_{1}, \ldots, \iota_{n}\right)$ is a major advantage when compared to the traditional country-level aggregate approach. As is done in recent studies in the context of human development measurement (see Grimm, Harttgen, Klasen and Misselhorn 2008, Grimm et al. 2010, Harttgen and Klasen 2011a, 2011b, Permanyer 2013), the improvements distribution can be left as is to guide researchers and policy-makers about local patterns of improvement, inform about the extent of inequality and give clues to understand why underdevelopment prevails in certain areas and what perpetuates it. An illustration of this approach is shown in section 4. Alternatively, researchers or decision-makers might also be interested in international or other highly-aggregated level comparisons. In such a different context, it is also possible to aggregate the detailed information of the improvements distribution to obtain an overall improvement indicator. We now present standard 
procedures to generate such an aggregate measure.

In this context, we define an overall improvement index as a continuous non-trivial function $I:[A, B]^{n} \rightarrow \mathbb{R}$, where $I\left(\iota_{1}, \ldots, \iota_{n}\right)$ is a summary statistic of the levels of improvement of the different units of analysis we are taking into account for a given country (analogous assumptions are made in welfare economics, where social utility is regarded as a function of individual utility levels). We now state certain reasonable postulates for an overall improvement index.

Fixed Point (FP): For any $\iota \in[A, B], I(\iota, \ldots, \iota)=\iota$.

When the improvement levels of the different units of analysis are all the same, the overall improvement index takes on this common value. Therefore, $I$ can be seen as an averaging operator that allows comparisons between populations of different sizes - as opposed to what would happen with unnormalized indices.

Additive Decomposability (AD): For any $\left(\iota_{1}, \ldots, \iota_{n}\right),\left(\widetilde{\iota}_{1}, \ldots, \widetilde{\iota}_{n}\right) \in[A, B]^{n}$ such that $\left(\iota_{1}+\right.$ $\left.\widetilde{\iota}_{1}, \ldots, \iota_{n}+\widetilde{\iota}_{n}\right) \in[A, B]^{n}$ one has that $I\left(\iota_{1}+\widetilde{\iota}_{1}, \ldots, \iota_{n}+\widetilde{\iota}_{n}\right)=I\left(\iota_{1}, \ldots, \iota_{n}\right)+I\left(\widetilde{\iota}_{1}, \ldots, \widetilde{\iota}_{n}\right)$.

This axiom can be interpreted as follows. Suppose the variable we are using to measure improvements has two components. For instance, if one is measuring percentage of vaccinated children, then its two components can be percentage of vaccinated girls and the percentage of vaccinated boys. Then AD says that the sum of improvements based on the vectors $\left(\iota_{1}, \ldots, \iota_{n}\right)$ and $\left(\tau_{1}, \ldots, \widetilde{\iota}_{n}\right)$ (that is: the improvements in vaccination among girls and boys) is the same as the improvements based on the vector $\left(\iota_{1}+\widetilde{\iota}_{1}, \ldots, \iota_{n}+\widetilde{\iota}_{n}\right)$ (that is: the improvements in vaccinated children). In other words, this shows how to calculate overall improvements when we split the underlying indicator in different components. 
The following theorem shows that FP and AD identify a unique overall improvement index.

Theorem 2. An overall improvement index satisfies FP and AD if and only if it can be written as

$$
I\left(\iota_{1}, \ldots, \iota_{n}\right)=\frac{\sum_{i=1}^{n} w_{i} \iota_{i}}{\sum_{i=1}^{n} w_{i}}
$$

Proof: See the Appendix.

Theorem 2 presents two necessary and sufficient conditions to characterize our overall improvement index as the population-weighted arithmetic mean of individual-specific improvements. As is clear, $I\left(\iota_{1}, \ldots, \iota_{n}\right)$ takes values between $A$ and $B$. Among others, this index satisfies the attractive property of "Factor Decomposability" (see Chakravarty and Majumder 2005: 282-283), which implies that, as long as the different $\iota_{j}$ are not all equal to zero, the percent contribution of a unit of analysis $i$ to overall improvement levels can simply be computed as

$$
C_{i}:=100 \frac{w_{i} \iota_{i}}{\sum_{j=1}^{n} w_{j}\left|\iota_{j}\right|}
$$

Observe that the improvements in the denominator are in absolute terms to avoid compensations between positive and negative values in case the range of values of the $\iota_{j}$ includes negative numbers. In addition, a contribution $C_{i}$ is negative whenever the corresponding $\iota_{i}$ is negative. Finally, one has that $\sum_{i}\left|C_{i}\right|=100$.

\section{Inequality-sensitive overall improvement indices}

The overall improvement index characterized in Theorem 2 is attractive for its transparency and simplicity. In particular, the property of Factor Decomposability alluded to in 
the previous paragraph is particularly useful to pinpoint those administrative units leaping ahead or lagging behind in the pace of progress toward well-being. However, this way of aggregating individual-specific improvement levels does not address important distributional concerns. As has been argued elsewhere, in certain scenarios it might be attractive from an ethical point of view to reward those distributions that are more equally distributed (see Atkinson 1970, Chakravarty 1990). Unfortunately, the arithmetic mean is not inequality sensitive when averaging subgroup-specific information into an overall welfare assessment index (see Alkire and Foster 2010, Foster et al 2005, Seth 2009). In order to remedy this problem, these authors suggest using the family of generalized weighted means, which are defined as follows:

$$
\mu^{\theta}\left(a_{1}, \ldots, a_{n} ; \omega_{1}, \ldots, \omega_{n}\right):=\left\{\begin{array}{c}
\left(\sum_{i=1}^{n} \omega_{i} a_{i}^{\theta}\right)^{1 / \theta} \text { if } \theta \neq 0 \\
\prod_{i=1}^{n} a_{i}^{\omega_{i}} \text { if } \theta=0
\end{array}\right\}
$$

where $a_{1}, \ldots, a_{n}$ is a set of non-negative real numbers and $\omega_{i}$ is the weight attached to observation $i$, with $\sum_{i} \omega_{i}=1$. As is well-known, whenever $\theta<1$ (resp. $\theta>1$ ), the index is more sensitive to the lower (resp. upper) tail of the $\left(a_{1}, \ldots, a_{n}\right)$ distribution. In particular, when $\theta=2, \theta=1, \theta=0$ and $\theta=-1$ one gets the weighted quadratic, arithmetic, geometric and harmonic means respectively. When $\theta \rightarrow \infty, \mu^{\theta}\left(a_{1}, \ldots, a_{n} ; \omega_{1}, \ldots, \omega_{n}\right) \rightarrow$ $\max \left\{a_{1}, \ldots, a_{n}\right\}$ and when $\theta \rightarrow-\infty, \mu^{\theta}\left(a_{1}, \ldots, a_{n} ; \omega_{1}, \ldots, \omega_{n}\right) \rightarrow \min \left\{a_{1}, \ldots, a_{n}\right\}$. The axiomatic characterization of this measure has been presented elsewhere in different contexts (e.g.: Aczél 1966, Bossert et al. 2009, Chakravarty 2011), so we will not reproduce it here.

When it comes to implement the generalized means in our framework, attention must be paid to the fact that the arguments of $\mu^{\theta}$ must be non-negative (otherwise, the power functions would be ill-defined). Therefore, from now onwards we will restrict our attention to those improvement indices $\iota^{a}$ and $\iota^{s}$ whose range does not include negative numbers 
(that is: with $A \geq 0$ ). When this is the case (and only in this case), we simply define our inequality-sensitive overall improvement indices as

$$
I^{\theta}\left(\iota_{1}, \ldots, \iota_{n}\right):=\mu^{\theta}\left(\iota_{1}, \ldots, \iota_{n} ; w_{1} / \sum_{i=1}^{n} w_{i}, \ldots, w_{n} / \sum_{i=1}^{n} w_{i}\right)
$$

Observe that when $\theta=1, I^{\theta}\left(\iota_{1}, \ldots, \iota_{n}\right)$ coincides with the overall improvement index characterized in Theorem 2.

\section{Consistency between achievement and shortfall improvement}

Having defined overall achievement and shortfall improvement indicators, it seems natural to ask whether these measures will provide consistent rankings when comparing alternative states of affairs. When dealing with the analogous problem in the context of inequality measurement, different authors have followed alternative approaches. Erreygers (2009) adopts a particularly strong interpretation of the consistency condition when he examines whether there exist inequality indices for which shortfall inequality is exactly equal to achievement inequality (i.e.: if a generic inequality index is denoted by $D$, he imposes $D(\mathbf{x})=D(\mathbf{p})$, where $\mathbf{p}=U-\mathbf{x})$. Short thereafter, Lambert and Zheng (2011) imposed a weaker consistency requirement according to which if a country $A$ is ranked to be less unequal in attainments than country $B$, then country $A$ should also exhibit less inequality in shortfalls than country $B$ (formally: $\left.D\left(\mathbf{x}_{A}\right)<D\left(\mathbf{x}_{B}\right) \Leftrightarrow D\left(\mathbf{p}_{A}\right)<D\left(\mathbf{p}_{B}\right)\right)^{4}$. As we see the later approach as quite natural when imposing consistency requirements, it is the one we have implemented in this paper in the context of improvements in standard of living. However, the fact of having achievement-specific and shortfall-specific improvement functions forces us to introduce some changes to our consistency condition, which reads as follows.

4 Recently, Lasso de la Vega and Aristondo (2012) approached the problem from a completely different angle: they suggest to construct an averaging operator $\Phi(I(\mathbf{x}), I(\mathbf{p}))$, where $\Phi(.,$.$) is the generalized mean$ of two real numbers. The implementation of this approach in the context of improvements in standards of living is beyond the scope of this paper and might be attempted in future research. 
Achievement and Shortfall Consistency (AS): Let $\mathbf{x}, \mathbf{y}, \mathbf{z}, \mathbf{w} \in[L, U]^{n}$ be any achievement distributions and let $\mathbf{p}=U-\mathbf{x}, \mathbf{q}=U-\mathbf{y}, \mathbf{u}=U-\mathbf{z}, \mathbf{v}=U-\mathbf{w} \in[0, U-L]^{n}$ be the corresponding shortfall distributions. Then

$$
I^{\theta}\left(\iota^{a}(\mathbf{x}, \mathbf{y})\right)<I^{\theta}\left(\iota^{a}(\mathbf{z}, \mathbf{w})\right) \Leftrightarrow I^{\theta}\left(\iota^{s}(\mathbf{p}, \mathbf{q})\right)<I^{\theta}\left(\iota^{s}(\mathbf{u}, \mathbf{v})\right)
$$

In words, $\mathrm{AS}$ imposes that if a country $A$ is considered to have experienced less overall improvement in standard of living than another country $B$ when measured with achievement distributions, then country $A$ should also be considered to have experienced less overall improvement in standard of living than country $B$ when measured with the corresponding shortfall distributions.

Our main results in this section are as follows:

Theorem 3. Assume we are using the achievement and shortfall improvement indicators characterized in Theorem 1 and the overall improvement indicator $I^{\theta}$. Whenever $\alpha>1$ or $\beta<1$, it is always possible to find achievement and the corresponding shortfall distributions (i.e.: $\mathbf{x}, \mathbf{y}, \mathbf{z}, \mathbf{w} \in[L, U]^{n}$ and $\left.\mathbf{p}=U-\mathbf{x}, \mathbf{q}=U-\mathbf{y}, \mathbf{u}=U-\mathbf{z}, \mathbf{v}=U-\mathbf{w} \in[0, U-L]^{n}\right)$ such that $I^{\theta}\left(\boldsymbol{\iota}^{a}(\mathbf{x}, \mathbf{y})\right)<I^{\theta}\left(\boldsymbol{\iota}^{a}(\mathbf{z}, \mathbf{w})\right)$ and $I^{\theta}\left(\boldsymbol{\iota}^{s}(\mathbf{p}, \mathbf{q})\right)>I^{\theta}\left(\boldsymbol{\iota}^{s}(\mathbf{u}, \mathbf{v})\right)$.

Proof: See the Appendix.

Corollary 1. When using the achievement and shortfall improvement indicators characterized in Theorem 1 and the overall improvement indicator $I^{\theta}$, the consistency condition AS holds if and only if $\alpha=\beta=1$.

Proof: See the Appendix.

Observe that whenever $\alpha=\beta=1$, one has that $\boldsymbol{\iota}^{a}(\mathbf{x}, \mathbf{y})=\boldsymbol{\iota}^{s}(\mathbf{p}, \mathbf{q})$, so $I^{\theta}\left(\iota^{a}(\mathbf{x}, \mathbf{y})\right)=$ $I^{\theta}\left(\boldsymbol{\iota}^{s}(\mathbf{p}, \mathbf{q})\right)$. Therefore, the only case in which the achievement and shortfall overall improve- 
ment measures characterized in this paper can rank alternative states of affairs consistently is the trivial case where both measures are exactly the same. Any departure from this trivialcase scenario (i.e.: if one lets $\iota^{a}(x, y)$ and $\iota^{s}(p, q)$ be different functions) leads to a violation of the consistency condition. Given the regulating role of $\alpha$ and $\beta$ regarding compliance with the Upward Sensitivity axiom, we can say that the later axiom is at odds with the axiom of Achievement and Shortfall Consistency.

\section{Empirical illustration}

In September 2000, the United Nations presented the Millennium Declaration, a milestone in international cooperation inspiring development efforts in order to improve the living conditions of millions of people around the world. As a result of the Millennium Declaration, all 193 United Nations member states agreed to achieve a series of time-bound targets -with a deadline of 2015- widely known as the 'Millennium Development Goals' (henceforth MDGs, see www.un.org/millenniumgoals). One of those goals - MDG \#4 - prompts countries all over the world to reduce child mortality. Clearly, this is a health outcome that can a priori be approached from two angles: the shortfall perspective (i.e.: reduce child mortality) or the attainment one (i.e.: increase child survivorship). While the official MDG \#4 is stated in terms of shortfalls ${ }^{5}$, one might legitimately wonder whether child health improvements will be consistent when assessed via the shortfall and attainment perspectives respectively. For this purpose, in this section we use census microdata from five African countries to assess the levels of child health improvement over time using the overall attainment and shortfall indices introduced in this paper.

5 MDG \#4 prompts countries to reduce by two thirds, between 1990 and 2015, the under-five mortality rate. 


\subsection{Data and indicators}

In order to construct child health indicators at local administrative levels we use census microdata samples from the Integrated Public Use Microdata Series database (IPUMS, see https://international.ipums.org/international) for the following countries: Malawi, Mali, Morocco, Rwanda and South Africa. Unfortunately, African countries available in the IPUMS database could not be included in our analysis, either because they lacked the appropriate variables to construct our health indicators or because the administrative units in the respective census years experienced big changes that made their monitoring over time extremely difficult.

The geographical detail available for each country is not uniform, as it depends on the density of the sample size (typically between $5 \%$ and $10 \%$ ), the distribution of the population and the way in which administrative units are defined for each country (see Table 1). For the case of Rwanda data are only available at the first administrative level (i.e.: the Province level), while for Mali and South Africa indicators can be computed at the third administrative level (i.e.: districts and municipalities, respectively). For Morocco and Malawi, indicators can be computed at the second administrative level (the specific name varies with each country). In cases where the corresponding statistical agencies permits access to complete census microdata files, it would be possible to extend the analysis presented in this paper to even lower levels with increasingly greater geographical detail.

[[[Table 1]]]

In order to measure child health outcomes for administrative unit $i$, we simply compute the percentage of surviving children born to women in that administrative unit between 
ages 20-39, which will be denoted by $P_{i}$. This indicator is particularly suitable for small size populations and has been used among other things to describe the socio-demographic characteristics of indigenous populations in Latin America (see ECLAC 2010) and to explore the distribution of human development levels with high geographical detail (see Permanyer 2013). Clearly, $P_{i}$ is an attainment indicator; its corresponding shortfall version is defined as $Q_{i}:=100-P_{i}$ (i.e.: the percentage of non-surviving children born to women between ages 20-39).

\subsection{Empirical results}

Figures 1 to 5 show the evolution of the distributions of child survivorship percentages of the corresponding administrative units for the different countries included in our analysis between two consecutive census years. The availability of such highly detailed data is a clear improvement with respect to the classical approach in which the values of the indicators of interest are only reported at high aggregation levels. Despite the reduced number of countries, the diversity of observed patterns is remarkable. For the case of Malawi (Fig. 1), the distribution of 1998 shows less dispersion and a higher average value when compared to the distribution of 1987. For the other countries, the shapes of the different density functions do not change substantially over time (roughly speaking: one seems to have been obtained from the other after a given translation). For the cases of Mali and Morocco there is an overall improvement over time (quite small for Morocco, see Figures 2 and 4) and for Rwanda and South Africa, there is an overall deterioration over time (see Figures 3 and 5$)^{6}$. The overall deterioration observed in Rwanda can be attributed to the massive killings that took place in the country in 1994, while the deterioration observed in South Africa - which

6 In this context, when we speak about overall 'improvement' or 'deterioration' we just refer to the general shape and position of the respective density functions, not to the specific changes observed for each administrative unit (which can not be inferred from that information only). 
is in line with official figures of declining life expectancy reported in that country - might be attributed to a large extent to the high prevalence of HIV/AIDS.

\section{[[[Figures 1-5]]]}

The density functions shown in Figures 1-5 only show the marginals of a distribution of paired data (that is: the achivement distributions in times $T_{1}$ and $\left.T_{2}:\left\{\left(x_{i}, y_{i}\right)\right\}_{1 \leq i \leq n}\right)$. However, these marginal distributions are not informative on the specific patterns of change over time of the different administrative units we are working with ${ }^{7}$. In order to show these administrative unit level improvements explicitly, Figure 6 plots the corresponding densities associated to the values of $\left(\iota_{1}, \ldots, \iota_{n}\right)$ when $\alpha=\beta=1, A=0$ and $B=1$ for the five countries studied in this section. Recall that when $\alpha=\beta=1, \iota^{a}\left(x_{i}, y_{i}\right)=\iota^{s}\left(p_{i}, q_{i}\right)$, so the attainment and shortfall distributions are exactly the same (later in this section we will explore the extent to which the attainment and shortfall distributions differ when we choose values for $\alpha$ and $\beta$ other than 1). Values of $\iota_{i}$ above (resp. below) 0.5 indicate that an actual improvement (resp. worsening) in child survivorship percentages has taken place in administrative unit i. As can be seen in Figure 6, the distribution of administrative units' child survivorship improvements over time has been quite different for the countries included in this study. At one extreme we have the cases of Rwanda and South Africa, where most administrative units have experienced deteriorations in child survivorship percentages. At the other extreme, most administrative units in Mali and Malawi have experienced improvements over time, as the $\iota_{i}$ values tend to be well above the threshold of 0.5. Somewhere in between we have the

7 To illustrate: assume, without loss of generality, that a distribution of achievements is ordered $\left(x_{1} \leq \ldots \leq\right.$ $\left.x_{n}\right)$. The hypothetical distributions of paired data $\left\{\left(x_{i}, y_{i}=x_{i}\right)\right\}_{1 \leq i \leq n}$ and $\left\{\left(x_{i}, y_{i}=x_{n-i+1}\right)\right\}_{1 \leq i \leq n}$ have exactly the same marginal distributions but the individual-level improvements are completely different in the two cases (there are no changes whatsoever in the first one and extreme changes are observed in the second one). 
case of Morocco, with a much more concentrated distribution around the value of 0.502 . In this case, 3 out of 4 administrative units have experienced slight improvements in child's health over time, while the opposite has been observed for the remaining ones.

\section{[[[Figure 6]]]}

The results shown in Figure 6 suggest that achievement distributions have generally improved over time for Mali and Malawi and deteriorated for Rwanda and South Africa. In this context, one might want to be more precise and quantify the extent of overall improvement in order to know how child's health has evolved over time. Table 2 shows the values of $I^{\theta}\left(\iota^{a}(\mathbf{x}, \mathbf{y})\right)$ and $I^{\theta}\left(\iota^{s}(\mathbf{p}, \mathbf{q})\right)$ for different specifications of parameters $\alpha, \beta$ and $\theta$. As can be seen, higher values of $\alpha$ tend to increase the values of $I^{\theta}\left(\iota^{a}(.,).\right)$ for the cases of Malawi, Mali and Morocco and decrease it for Rwanda and South Africa. Alternatively, as $\beta$ decreases, the behavior of $I^{\theta}\left(\iota^{s}(.,).\right)$ is unclear for the different countries considered here. This suggests that the behavior of $I^{\theta}\left(\iota^{a}(.,).\right)$ does not mirror that of $I^{\theta}\left(\iota^{s}(.,).\right)$ as $\alpha$ increases and $\beta$ decreases, even if in both cases improvements at higher achievement and lower shortfall levels are being rewarded. Regarding the ranking of countries in terms of overall improvements, Mali, Malawi and Morocco unambiguously rank in the first, second and third positions respectively no matter what choice we make about $\alpha, \beta$ and $\theta$. Alternatively, the relative position of Rwanda vis-à-vis South Africa depends on the choice of those parameters. For instance, when $\alpha=5, \theta=1$, Rwanda is ranked above South Africa, but such ranking is reversed if one chooses $\alpha=\theta=1$. Table 2 also shows the values of the Gini index of the achievement and shortfall improvement distributions $\left(\iota_{1}, \ldots, \iota_{n}\right)$ for different choices of $\alpha$ and $\beta$. As can be seen, the spread of those improvement distributions is quite small (the Gini index is never higher than 0.05). The distributions that tend to be more 
spread out are those of Mali, Malawi and South Africa, while Morocco stands out as the most concentrated distribution. In general, the spread of the distributions tend to increase with $\alpha$, but the relationship with the values of $\beta$ is unclear.

[[[Table 2]]]

We will finally explore empirically the extent to which the assessment of improvement levels is consistent when using the shortfall and achievement perspectives. For that purpose, within each country we compare the administrative units ranking that is obtained using $\iota^{a}(\mathbf{x}, \mathbf{y})$ with the ranking derived from the values of $\iota^{s}(\mathbf{p}, \mathbf{q})$. Table 3 shows the values of Kendall's tau coefficient ${ }^{8}$ (henceforth $\tau$ ) associated to the values of those achievement and shortfall indicators for alternative choices of $\alpha$ and $\beta$. The definition of that statistic fits perfectly in our framework, since our consistency axiom (AS) precisely demands that the same set of individuals is coherently ranked by alternative measures. It turns out that in all comparisons considered in this paper except for one ${ }^{9}$ the administrative units rankings that are obtained from the values of $\boldsymbol{\iota}^{a}(\mathbf{x}, \mathbf{y})$ and $\boldsymbol{\iota}^{s}(\mathbf{p}, \mathbf{q})$ are not completely consistent, that is: there exist couples of administrative units whose relative ranking is reversed when using the achievement and shortfall indicators. When this happens, the corresponding $\tau$ is strictly smaller than 1. Despite not being completely consistent, the association between both rankings is quite high in most countries (the different values of $\tau$ tend to be higher than 0.8). For the case of South Africa, however, there are certain choices of $\alpha$ and $\beta$

8 Let $\left(x_{1}, y_{1}\right), \ldots,\left(x_{n}, y_{n}\right)$ be a set of observations of the joint random variables $X$ and $Y$. Assuming there are no ties, Kendall's tau is defined as $\tau:=(C-D) /(n(n-1) / 2)$, where $C$ (resp. $D$ ) is the number of concordant (resp. discordant) pairs of observations and $n(n-1) / 2$ is the total number of pair combinations. When all couples of observations are consistently ranked by $X$ and $Y, \tau=1$ and when all couples of observations are inconsistently ranked, $\tau=-1$.

9 The exception to the rule is found in Rwanda when $\alpha=5$ and $\beta=1 / 2$. In that case, all couples of administrative units are consistently ranked by $\iota^{a}$ and $\iota^{s}$. 
for which $\tau \simeq 0.7$, that is: many couples of administrative units (up to 30\%) are not ranked consistently according to $\iota^{a}$ and $\iota^{s}$. Therefore, our assessments of the child health improvements experienced in South African municipalities can differ to a great extent when using achievement or shortfall indicators.

[[[Table 3]]]

\section{Summary and concluding remarks}

In this paper we have presented new indices of improvements in standards of living that address two important issues which have been ignored so far in the literature: the problem of "consistently measuring achievement and shortfall improvement" and the problem of "unobserved heterogeneity". Given the bounded nature of virtually all standard of living indicators, it is possible to measure their improvements over time on the basis of the levels of achievement or on the basis of the corresponding shortfalls with respect to the upper bound. Integrating both approaches into a common framework, we have proposed the corresponding achievement and shortfall improvement indices and characterized them axiomatically. In a way, our improvement indices are reminiscent of the directional income mobility indices suggested by Fields and Ok (1999) adapted to the case where the variable of interest is bounded.

We argue that achievement and shortfall improvements are two sides of the same coin and that it is important to check whether both sides are measured in a consistent way. Such consistency can be imposed in different ways. A strong requirement to fulfill the consistency condition is to impose that both achievement and shortfall improvement indices take exactly the same values. A weaker requirement simply states that the orderings derived from the values of the indices have to be the same. It turns out that even when starting from the weaker 
requirement, the only achievement and shortfall improvement indices that rank alternative states of affairs in a consistent way are those that take exactly the same values. Or the other way around: it is only when achievement and shortfall improvement indices are the same that they are able to rank alternative states of affairs consistently. As shown in our analysis, the only way in which achievement and shortfall improvement indices can be exactly the same is to get rid of the 'Upward Sensitivity' axiom, a common requirement in the literature suggested by Sen (1981) which rewards those improvements occurring at higher achievement (or lower shortfall) levels. This somewhat discouraging result is in line with the findings reported by Lambert and Zheng (2011) in the context of consistent achievement-shortfall inequality measurement. Given the fact that the achievement and shortfall approaches are not perfectly complementary (i.e.: the orderings derived from one approach can not be deduced from the orderings of the other), it becomes necessary to give them separate and careful attention.

The improvement indices proposed in the literature so far have only shown results at the country level, therefore hiding potentially large internal inequalities that might exist at lower aggregation levels. It is in this context that this paper also aims to make a contribution: collecting information at arbitrary small levels of aggregation we are able to uncover unobserved heterogeneity, complementing traditional country-level analysis and taking into account the entire distribution of local-level improvements over time. With such fine-grained information, one can either investigate the distribution of improvements as is (i.e.: without attempting to generate higher level aggregates) or investigate the effect that the inequality of the local-level improvements distribution has on the overall improvement at the country-level (an approach that is based on the proposal made by Atkinson 1970 in which social welfare is penalized by existing inequality levels). Both perspectives - which have been implemented 
here using standard techniques from welfare analysis - can be particularly useful for policy makers in order to assess improvement levels of standard of living with greater accuracy and reliability.

We have empirically illustrated our methodology exploring the evolution of child survivorship percentages in five African countries after two consecutive census rounds. Among other things, our results indicate that within each country, the corresponding administrative units rankings derived from the values of our achievement and shortfall indicators are not always completely consistent (that is: there always exist some couples of administrative units which are inconsistently ranked with both kind of indicators). Even if both rankings tend to be highly correlated for most countries studied here, in the case of South Africa there are as many as $30 \%$ of couples of municipalities that are inconsistently ranked by certain specifications of our achievement and shortfall improvement indicators. Therefore, consistency is not only a matter of theoretical import but it is also a problem that can be encountered in practice to a large extent.

\section{Appendix}

\section{Proof of Theorem 1.}

Theorem 1 gives the necessary and sufficient conditions that characterize our achievement and shortfall improvement indices. Here we will only show the "achievement part" of the proof; the shortfall version is extremely similar and will not be reproduced here to avoid redundancies. However, it is available upon request to any interested reader.

It is straightforward to prove that the function $\iota^{a}(x, y)=(B-A)\left[y^{\alpha}-x^{\alpha}\right] / 2\left(U^{\alpha}-L^{\alpha}\right)+$ $(A+B) / 2$ with $\alpha \geq 1$ satisfies $\mathrm{CN}, \mathrm{MN}, \mathrm{HM}$, US, WA and NM, so we will just prove that 
whenever a function $m:[L, U]^{2} \rightarrow \mathbb{R}$ satisfies all these axioms, then it must be necessarily equal to $\iota^{a}(x, y)$.

Consider any real-valued function $m:[L, U]^{2} \rightarrow \mathbb{R}$ and any $(x, y) \in[L, U]^{2}$. By WA one has that

$$
\begin{aligned}
& m(x, x)+m(x, y)=\varphi(x, y) \\
& m(x, y)+m(y, y)=\varphi(x, y)
\end{aligned}
$$

for some function $\varphi:[L, U]^{2} \rightarrow \mathbb{R}$. From equations $(\mathrm{A} 1)$ and (A2) one deduces that $m(x, x)=$ $c \forall x \in[L, U]$ for some real constant $c$.

Consider now any triple $(x, y, z) \in[L, U]^{3}$. Again, by WA one has that

$$
\begin{aligned}
& m(x, y)+m(y, z)=\varphi(x, z) \\
& m(x, x)+m(x, z)=\varphi(x, z)
\end{aligned}
$$

From equations (A3) and (A4) one deduces that

$$
m(x, y)=m(x, z)-m(y, z)+m(x, x)=m(x, z)-m(y, z)+c
$$

Let $z$ be equal to some arbitrary constant $d \in[L, U]$ and let $f(x):=m(x, d)$ for any $x \in[L, U]$. Then (A5) can be simply written as

$$
m(x, y)=f(x)-f(y)+c
$$

By $\mathrm{CN}$ and MN, $f(x)$ must be a continuous decreasing function of $x$. HM implies that for all $x, y, u, v \in[L, U]$ and all $\lambda>0$ such that $\lambda x, \lambda y, \lambda u, \lambda v \in[L, U]$

$$
f(x)-f(y)+c=f(u)-f(v)+c \Leftrightarrow f(\lambda x)-f(\lambda y)+c=f(\lambda u)-f(\lambda v)+c
$$


Following Eichhorn and Gleissner (1988:25), equation (A7) implies that

$$
f(\lambda x)-f(\lambda y)=F(f(x)-f(y), \lambda)
$$

for some continuous function $F(.,$.$) . By equation (A8) we have that$

$$
f(\lambda y)-f(\lambda z)=F(f(y)-f(z), \lambda)
$$

and

$$
f(\lambda x)-f(\lambda z)=F(f(x)-f(z), \lambda)
$$

Adding (A9) to (A8) we get

$$
f(\lambda x)-f(\lambda z)=F(f(x)-f(y), \lambda)+F(f(y)-f(z), \lambda)
$$

Since $f$ is continuous and non-constant,

$$
f(x)-f(y)=: X, f(y)-f(z)=: Y, f(x)-f(z)=: X+Y
$$

lie in a proper interval $I \subseteq \mathbb{R}$ containing zero. The right hand sides of (A10) and (A11) have to be equal, so using the notation introduced in (A12) we have that $F$ satisfies the Cauchy equation

$$
F(X+Y, \lambda)=F(X, \lambda)+F(Y, \lambda)
$$

for all $X, Y, X+Y \in I$. We are now going to extend this result to the set of non-negative real numbers $\mathbb{R}_{+}$.

Let $s:=\max _{x \in I}\{x\}$ and let $c \in \mathbb{R}_{+}$. We can write $c=p / q$, for some $p, q \in[0, \min \{1, s\}] \subset$ $I$. Let's define $H(c, \lambda):=F(p, \lambda) / F(q, \lambda)$ for $c>0$ and $H(0, \lambda):=F(0, \lambda)$. Since $F(0, \lambda)=$ $F(0+0, \lambda)=F(0, \lambda)+F(0, \lambda)$, one has that $H(0, \lambda)=0$. Since $F$ satisfies the Cauchy equation (A13), it is straightforward to prove that $F(k X, \lambda)=k F(X, \lambda)$ for all $k \in[0, \min \{1, s\}]$ 
(see Aczél 1966:34). Therefore, the function $H$ is well defined: if one has that $c_{1}=c_{2} \in \mathbb{R}_{+}$, then it is possible to write $c_{1}=p_{1} / q_{1}$ and $c_{2}=\left(k p_{1}\right) /\left(k q_{1}\right)$ for some $p_{1}, q_{1}, k \in[0, \min \{1, s\}]$, so that

$$
H\left(c_{2}, \lambda\right)=\frac{F\left(k p_{1}, \lambda\right)}{F\left(k q_{1}, \lambda\right)}=\frac{k F\left(p_{1}, \lambda\right)}{k F\left(q_{1}, \lambda\right)}=\frac{F\left(p_{1}, \lambda\right)}{F\left(q_{1}, \lambda\right)}=H\left(c_{1}, \lambda\right)
$$

Let us now consider any $c_{1}, c_{2} \in \mathbb{R}_{+}$. It is possible to write $c_{1}=p_{1} / q_{1}$ and $c_{2}=p_{2} / q_{2}$ for some $p_{1}, q_{1}, p_{2}, q_{2} \in[0, \min \{1, s\}]$. Now, one has that

$$
H\left(c_{1}+c_{2}, \lambda\right)=H\left(\frac{p_{1} q_{2}+p_{2} q_{1}}{q_{1} q_{2}}, \lambda\right)=\frac{F\left(p_{1} q_{2}+p_{2} q_{1}, \lambda\right)}{F\left(q_{1} q_{2}, \lambda\right)}
$$

Given the fact that $p_{1} q_{2}, p_{2} q_{1} \in(0, \min \{1, s\}] \subset I$, the Cauchy equation (A13) applies, so the last expression can be written as

$$
\frac{F\left(p_{1} q_{2}, \lambda\right)}{F\left(q_{1} q_{2}, \lambda\right)}+\frac{F\left(p_{2} q_{1}, \lambda\right)}{F\left(q_{1} q_{2}, \lambda\right)}=\frac{F\left(p_{1}, \lambda\right)}{F\left(q_{1}, \lambda\right)}+\frac{F\left(p_{2}, \lambda\right)}{F\left(q_{2}, \lambda\right)}=H\left(c_{1}, \lambda\right)+H\left(c_{2}, \lambda\right)
$$

Hence, $H$ satisfies the Cauchy equation for any positive number $c_{1}, c_{2} \in \mathbb{R}_{+}$. Moreover, by Continuity of $F, H$ must be continuous too at least in a single point. Applying the characterization result found in Aczél (1966: 34), one must have that $H(t, \lambda)=t \alpha(\lambda)$ for some continuous function $\alpha$. Since $H$ is an extension of $F$, one must also have that

$$
F(t, \lambda)=\alpha(\lambda) t
$$

Therefore,

$$
f(\lambda x)-f(\lambda y)=\alpha(\lambda)(f(x)-f(y))
$$

for all $x, y, \lambda x, \lambda y \in[L, U]$. The solution to this functional equation is also well-known (see Aczél 1988):

$$
f(x)=\left\{\begin{array}{c}
C x^{r}+D \\
P \ln (x)+Q
\end{array}\right\}
$$


where $C, D, P, Q, r$ are arbitrary real constants. Imposing $\mathrm{MN}$ and $\mathrm{NM}$ on equations (A6) and (A19) we must conclude that either

$$
\begin{gathered}
m(x, y)=(B-A) \frac{y^{r}-x^{r}}{2\left(U^{r}-L^{r}\right)}+\frac{A+B}{2} \text { or } \\
m(x, y)=(B-A) \frac{\ln (y)-\ln (x)}{2(\ln (U)-\ln (L))}+\frac{A+B}{2}
\end{gathered}
$$

for some parameters $A, B, r$.

Let $L \leq z<w \leq U$ and consider any $\delta>0$ such that $z+\delta, w+\delta \in[L, U]$. Imposing US on equation (A20) one must have that

$$
(z+\delta)^{r}-z^{r} \leq(w+\delta)^{r}-w^{r}
$$

Clearly, (A22) is only satisfied when the function $x^{r}$ is convex, that is, when $r \geq 1$. On the other hand, the function shown in equation (A21) does not satisfy US. This completes the proof of Theorem 1 .

\section{Q.E.D.}

\section{Proof of Theorem 2.}

It is straightforward to prove that $I\left(\iota_{1}, \ldots, \iota_{n}\right)=\left(\sum_{i=1}^{n} w_{i} \iota_{i}\right) / \sum_{i=1}^{n} w_{i}$ satisfies $\mathrm{FP}$ and AD. We will only prove the reverse implication.

Let $\left(x_{1}, \ldots, x_{n}\right) \in[A, B]^{n}$ and let $c \in[A, B]$ be a real constant such that $c+x_{i} \in[A, B]$ for all $i$. By $\mathrm{AD}$ one has that

$$
I\left(x_{1}+c, \ldots, x_{n}+c\right)=I\left(x_{1}, \ldots, x_{n}\right)+I(c, \ldots, c)
$$

By FP, the last equation can be rewritten as

$$
I\left(x_{1}+c, \ldots, x_{n}+c\right)=I\left(x_{1}, \ldots, x_{n}\right)+c
$$


According to equation (A24), for any $i, 1 \leq i \leq n$, we can write

$$
I\left(A, \ldots, A, A+x_{i}, A, \ldots, A\right)=I\left(0, \ldots, 0, x_{i}, 0, \ldots, 0\right)+A=f_{i}\left(x_{i}\right)+A
$$

for some function $f_{i}:[A, B] \rightarrow \mathbb{R}$. Applying $\mathrm{AD}$, one has that

$$
I\left(x_{1}+A, A, A, \ldots, A\right)+I\left(A, x_{2}+A, A, \ldots, A\right)=I\left(x_{1}+2 A, x_{2}+2 A, 2 A, \ldots, 2 A\right) .
$$

Applying (A24), equation (A26) can be rewritten as

$$
f_{1}\left(x_{1}\right)+f_{2}\left(x_{2}\right)=I\left(x_{1}, x_{2}, 0, \ldots, 0\right)
$$

Repeating this procedure one has that

$$
I\left(x_{1}, \ldots, x_{n}\right)=\sum_{i=1}^{n} f_{i}\left(x_{i}\right)
$$

Applying $\mathrm{AD}$, for any $i, 1 \leq i \leq n$ one has that

$f_{i}\left(x_{i}+y_{i}\right)=I\left(0, \ldots, 0, x_{i}+y_{i}, 0, \ldots, 0\right)=I\left(0, \ldots, 0, x_{i}, 0, \ldots, 0\right)+I\left(0, \ldots, 0, y_{i}, 0, \ldots, 0\right)=f_{i}\left(x_{i}\right)+f_{i}\left(y_{i}\right)$

Therefore, $f_{i}($.$) satisfies the Cauchy equation for all x_{i}, y_{i} \in[A, B]$ such that $x_{i}+y_{i} \in$ $[A, B]$. Using the same kind of arguments as the ones shown between equations (A13) and (A17), it is straightforward to extend equation (A29) to the set of all real numbers. Since overall improvement functions $I\left(x_{1}, \ldots, x_{n}\right)$ are assumed to be continuous, the $f_{i}($.$) must be$ continuous too, so we can apply the characterization result of Aczél (1966: 34) according to which $f_{i}\left(x_{i}\right)=p_{i} x_{i}$ for some constant $p_{i} \in \mathbb{R}$. Therefore, equation (A28) becomes

$$
I\left(x_{1}, \ldots, x_{n}\right)=\sum_{i=1}^{n} p_{i} x_{i}
$$

According to FP, $I(c, \ldots, c)=c \sum_{i} p_{i}=c$, so $\sum_{i} p_{i}=1$. This completes the proof of Theorem 2 . 
Q.E.D.

\section{Proof of Theorem 3.}

The basic idea of the proof is as follows: we will show that whenever $\alpha>1$ or $\beta<$ 1, we are always able to find some couples of achievement distributions $(\mathbf{x}, \mathbf{y})$ and $(\mathbf{z}, \mathbf{w})$ whose respective rankings in terms of improvements in achievements is not consistent when compared with respect to the ranking in terms of improvements in shortfalls obtained from the corresponding shortfall distributions $(\mathbf{p}, \mathbf{q})$ and $(\mathbf{u}, \mathbf{v})$.

Assume that each individual experiences the same improvement over time. Since $I^{\theta}$ satisfies FP, there exist real numbers $x, y \in[L, U]$ and $p=U-x, q=U-y \in[0, U-$ $L]$ such that $I^{\theta}\left(\iota^{a}(\mathbf{x}, \mathbf{y})\right)=\iota^{a}(x, y)$ and $I^{\theta}\left(\iota^{s}(\mathbf{p}, \mathbf{q})\right)=\iota^{s}(p, q)$, where $\iota^{a}$ and $\iota^{s}$ are the achievement and shortfall improvement functions characterized in Theorem 1. Pick any $\left(x_{0}, y_{0}\right) \in[L, U]^{2}$ with $x_{0} \neq y_{0}$ and consider the level contour of $\iota^{a}(x, y)$ passing through that point: $l_{\left(x_{0}, y_{0}\right)}^{a}:=\left\{(x, y) \in[L, U]^{2} \mid \iota^{a}(x, y)=\iota^{a}\left(x_{0}, y_{0}\right)\right\}$. After some basic algebraic manipulation, it is straightforward to write a generic member of the level contour $l_{\left(x_{0}, y_{0}\right)}^{a}$ as $\left(x, \eta_{a}(x)\right)$, where

$$
\eta_{a}(x)=\left(y_{0}^{\alpha}-x_{0}^{\alpha}+x^{\alpha}\right)^{1 / \alpha}
$$

Differentiating this function, one gets

$$
\eta_{a}^{\prime}(x)=\frac{x^{\alpha-1}}{\left(y_{0}^{\alpha}-x_{0}^{\alpha}+x^{\alpha}\right)^{1-1 / \alpha}}
$$

so one has that

$$
\eta_{a}^{\prime}\left(x_{0}\right)=\left(\frac{y_{0}}{x_{0}}\right)^{1-\alpha}
$$

We are now going to do the same for our shortfall improvement function. Observe first that $\iota^{s}$ can also be written as a function depending on achievements: $\psi(x, y):=\iota^{s}(U-$ 
$x, U-y)=\iota^{s}(p, q)$. As before, consider the level contour of $\psi(x, y)$ passing through $\left(x_{0}, y_{0}\right) \in$ $[L, U]^{2}$, that is: $l_{\left(x_{0}, y_{0}\right)}^{s}:=\left\{(x, y) \in[L, U]^{2} \mid \psi(x, y)=\psi\left(x_{0}, y_{0}\right)\right\}$. Manipulating algebraically, we can rewrite a generic member of $l_{\left(x_{0}, y_{0}\right)}^{s}$ as $\left(x, \eta_{s}(x)\right)$, where

$$
\eta_{s}(x)=U-\left[(U-x)^{\beta}-\left(U-x_{0}\right)^{\beta}+\left(U-y_{0}\right)^{\beta}\right]^{1 / \beta}
$$

Differentiating this function, one gets

$$
\eta_{s}^{\prime}(x)=\frac{\left[(U-x)^{\beta}-\left(U-x_{0}\right)^{\beta}+\left(U-y_{0}\right)^{\beta}\right]^{1 / \beta-1}}{(U-x)^{1-\beta}}
$$

so one has that

$$
\eta_{s}^{\prime}\left(x_{0}\right)=\left(\frac{U-y_{0}}{U-x_{0}}\right)^{1-\beta}
$$

As can be seen in equations (A33) and (A36), whenever $x_{0} \neq y_{0}$ and $\alpha>1$ or $\beta<1$, one has that $\eta_{a}^{\prime}\left(x_{0}\right) \neq \eta_{s}^{\prime}\left(x_{0}\right)$. In words: the slopes of the level contours of the achievement and shortfall improvement functions passing through $\left(x_{0}, y_{0}\right)$ are not the same. Therefore, in a sufficiently small neighborhood of $\left(x_{0}, y_{0}\right)$ (for instance a ball of radius $r$ centered at that point $B\left(\left(x_{0}, y_{0}\right), r\right):=\left\{(x, y) \in[L, U]^{2} \mid d\left((x, y),\left(x_{0}, y_{0}\right)\right)<r\right\}$ where $d((.,),.(.,)$.$) is a$ distance function between two points in $\left.\mathbb{R}^{2}\right)$ it is possible to find some $\left(z_{0}, w_{0}\right) \in B\left(\left(x_{0}, y_{0}\right), r\right)$ such that $\iota^{a}\left(x_{0}, y_{0}\right)<\iota^{a}\left(z_{0}, w_{0}\right)$ and $\psi\left(x_{0}, y_{0}\right)>\psi\left(z_{0}, w_{0}\right)$. This argument is grafically illustrated in Figure A1.

\section{[[[Figure A1]]]}

Stated otherwise: whenever $\alpha>1$ or $\beta<1$, we have been able to find some achievements $\left(x_{0}, y_{0}\right),\left(z_{0}, w_{0}\right)$ whose ranking in terms of $\iota^{a}$ is reversed when comparing it with the ranking of the corresponding shortfalls $\left(U-x_{0}, U-y_{0}\right),\left(U-z_{0}, U-w_{0}\right)$ in terms of $\iota^{s}$. This completes the proof of Theorem 3 . 
Q.E.D.

\section{Proof of Corollary 1.}

According to Theorem 3, whenever $\alpha>1$ or $\beta<1$ AS does not hold. The only case which has not been examined is $\alpha=\beta=1$. In that case, one simply has that $\iota^{a}(x, y)=$ $\iota^{s}(p, q)=(B-A)[y-x] / 2(U-L)+(A+B) / 2$. When the achievement and shortfall improvement functions are the same, AS is trivially satisfied.

Q.E.D.

Acknowledgements: Financial support from the Spanish Ministry of Education "Juan de la Cierva" Research Grant Program and research projects ERC-2009-StG-240978 and ECO2010-21668-C03-02 is gratefully acknowledged.

\section{REFERENCES}

Aczél, J. Lectures on Functional Equations and their applications. Academic Press: New York; 1966.

Aczél, J. Cheaper by the dozen: Twelve functional equations and their applications to the laws of science and to measurement in economics, in: Eichhorn, W. (ed.), Measurement in Economics: Theory and Applications in Economic Indices, Heidelberg: Physica-Verlag; 1988.

Alkire, S., Foster, J. Designing the Inequality-Adjusted Human Development Index (IHDI). 2010, Human Development Research Paper; 2010/28; UNDP.

Atkinson, A. On the Measurement of Inequality. Journal of Economic Theory 1970; 2; 244-263.

Bossert, W., Chakravarty, S., D'Ambrosio, C. Multidimensional Poverty and material deprivation. ECINEQ WP 2009-129.

Chakravarty, S. and Mukherjee, D. Measuring improvement in well-being. Keio Economic Studies 1999; 36(2); 65-79. 
Chakravarty, S. and Majumder, A. Measuring human poverty: a generalized index and an application using basic dimensions of life and some antrhopometric indicators. Journal of Human Development 2005; 6(3); 275-299.

Chakravarty, S. Ethical social index numbers. Springer Verlag; 1990.

Chakravarty, S. A reconsideration of the tradeoffs in the new human development index. Journal of Economic Inequality 2011; 9; 471-474.

Clarke, P., Gerdtham, U., Johannesson, M. Bingerfors, K. and Smith, L. On the measurement of relative and absolute income-related health inequality. Social Science \& Medicine 2002; 55(11); 1923-1928.

Dasgupta, P. An Inquiry into the Sources of Well-being and Destitution. Oxford: Clarendon Press; 1993.

Dasgupta, P., Weale, M. On Measuring the Quality of Life. World Development 1992; $20 ; 119-131$.

Easterly, W. Life during growth. Journal of Economic Growth 1999; 4; 239-276.

ECLAC. Pueblos Indígenas y Afrodescendientes. Publication series by ECLAC's Population Division; 2010.

Erreygers, G. Can a single indicator measure both attainment and shortfall inequality? Journal of Health Economics 2009; 28; 885-893.

Eichhorn, W., Gleissner, W. The equation of measurement, in: Eichhorn, W. (ed.) Measurement in Economics: Theory and Applications in Economic Indices. Heidelberg: Physica-Verlag; 1988.

Fields, G., Ok,E. Measuring movement of incomes. Economica 1999; 66; 455-471.

Foster, J., Lopez-Calva, L., Szekely, M. Measuring the distribution of human development: methodology and an application to Mexico. Journal of Human Development $2005 ; 6 ; 5-29$.

Grimm, M., Harttgen, K., Klasen, S., Misselhorn, M. A Human Development Index by Income Groups. World Development 2008; 36 (12); 2527-2546.

Grimm, M., Harttgen K., Klasen, S., Misselhorn, M., Munzi, T., Smeeding, T. Inequality in Human Development: An Emprirical Assessment of 32 Countries. Social Indicators Research 2010; 97; 191-211.

Harttgen, K., Klasen, S. A Human Development Index by Internal Migration Status. Journal of Human Development and Capabilities 2011a; 12(3); 393-424. 
Harttgen, K., Klasen, S. A Household-based Human Development Index. World Development 2011b; 40(5); 878-899.

Kakwani, N. Performance in living standards: an international comparison. Journal of Development Economics 1993; 41; 307-336.

Lambert, P. and Zheng, B. On the consistent measurement of attainment and shortfall inequality. Journal of Health Economics 2011; 30; 214-219.

Lasso de la Vega, C. and Aristondo, O. Proposing indicators to measure achievement and shortfall inequality consistently. Journal of Health Economics 2012; 31; 578-583.

Majumder, A., Chakravarty, S. Achievement and improvement in living standards. Journal of Development Economics 1996; 50; 189-195.

Mazumdar, K. Measuring the well-beings of the developing countries: achievement and improvement indices. Social Indicators Research 1999; 47; 1 - 60.

Permanyer, I. Using Census Data to Explore the Spatial Distribution of Human Development. World Development 2013; 46; 1-13.

Sen, A. Public action and the quality of life in developing countries. Oxford Bulletin of Economics and Statistics 1981; 43; 287-319.

Sen, A. Inequality reexamined. Clarendon Press, Oxford; 1992.

Seth, S. Inequality, interactions and human development. Journal of Human Development and Capabilities 2009; 10; 375-396.

Tsui, K. Improvement indices of well-being. Social Choice and Welfare 1996; 13; 291303.

United Nations. The Millennium Development Goals Report 2010; 2010a.

United Nations. Assessing progress in Africa toward the Millennium Development Goals; $2010 b$.

United Nations. Charting the progress of the Millennium Development Goals in the Arab Region; 2010c.

United Nations. Achieving the MDGs in an era of global uncertainty: Asia and the Pacific Regional Report; 2010d.

United Nations. Avances en la sostenibilidad ambiental del desarrollo en Américana Latina; 2010e. 